\title{
Current Development of the Applications of Polymers in Gene Delivery
}

\author{
Huiming $\mathrm{Wu}^{1}$ \\ ${ }^{1}$ Department of Chemistry, Virginia Tech, Blacksburg, 24061, Virginia, US
}

\begin{abstract}
Gene delivery is one of the most important and efficient therapy methods for treating diseases, especially genetic diseases that challenge conventional treatment methods. However, to be efficient, gene delivery has a few requirements for the polymers to be applicable, including the reduction of cytotoxicity, the improvement of transfection efficiency, and the elimination of off-target effects. In this study, the common polymers applied in gene delivery is discussed in depth, regarding such requirements, as well as some of the limitations and potential improvements in gene delivery.
\end{abstract}

\section{Introduction}

Cancers and numerous diseases including genetic and viral diseases had a long history with human in terms of progressions from having no treatment methods to numerous therapies such as chemotherapy, radiation therapy and physical therapy. Commonly, chemotherapy and radiation therapy are combined to treat cancers which show a greater survival of a patient with esophageal cancer but with a cause of greater side effects. [1] Physical therapies involves the improvement of patients health after injuries and has low connection with treating diseases. Gene therapy is a new therapeutic method, but it shows people some important history for the development of this method. In 1963, Joshua Lederberg anticipated the polynucleotide could be grafted onto a virus DNA utilizing chemistry. Then, Rosenberg with his colleague started the first clinical study around gene transfer. Metastatic melanoma patients' lymphocytes were obtained and neomycin resistance gene was transferred and cultivated in vitro. After, the neomycin was transferred back to the respective patients. Rosenburg et al observed that the gene modified cells were present and no side effects observed. [2] This demonstration of gene therapy shows a promising potential, safeness, and effectiveness which lead to more studies around the subject. [3] As the current decades progresses, gene therapy progresses to the pictures that people see today.

Gene delivery or gene therapy, has become an increasingly interested and effective method for treating diseases, genetic diseases to be specific, in the recent decades. [4] Gene delivery has become a treatment method for treating the diseases that the conventional drug delivery systems have trouble dealing with, such as the first human immunodeficiency virus (HIV) patient, Timothy Ray Brown, recovered from HIV by gene delivery. Although conventional drug delivery systems show effectiveness in treating a large portion of diseases caused by numerous factors such as heart disease, the diseases caused by only the absence or extra of proteins is most efficiently treated with gene therapy. The diminishing returns for the time and money investment in conventional drug for treating genetic disease prompted scientists to invest in gene delivery which shows greater potential in treating such disease in general. Besides genetic diseases, gene delivery is also really promising in treating other diseases such as viral infection and cancers. [5] Gene delivery is different from conventional drug delivery regarding the properties of the materials that have therapeutic effect, such as hydrophobicity, and the delivery vehicle.

Gene delivery is a delivery method that involves the delivery of genomic materials such as small interfering RNA (siRNA) and DNA, utilizing viral vectors or nonviral vectors. Although there are two categories for gene delivery which correspond to germline and somatic delivery system, only somatic delivery system is appropriate because of the unethicality of the germline delivery system. Viral vector is a delivery of the genomic materials, typically commonly utilizing adenovirus as a vehicle for packing. [6] Viral vectors essentially utilize the cell entry mechanism of viruses to deliver siRNA to bind with messenger RNA (mRNA) to silence a protein expression. The siRNA is able to be replicated in the host cell, DNA delivery is mainly utilized to express a certain protein that the host might not have, for treating the specific disease. However, due to the toxicity, potential replication, cost, and carrying capacity of viral vectors, the gene delivery utilizing nonviral vectors are more promising. [6] Non-viral vector, as is sounds, uses polymers as delivery vehicle other than viruses. As mentioned above, viral vector has disadvantages are the potential genotoxicity in the process of inserting the genetic materials of the virus into the cell genome. [4] Thus, non-viral vector is the 
main interest of this paper as it involves the application of various polymers.

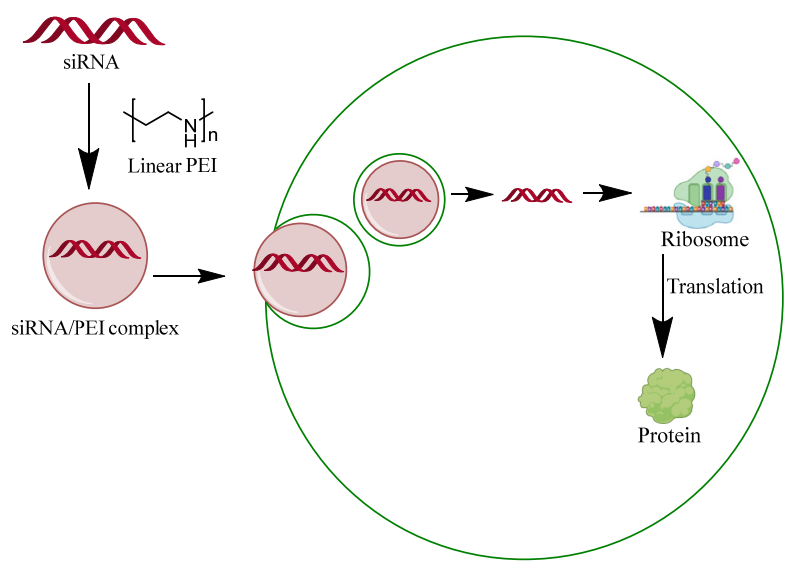

Figure 1. Overview of polymer-mediated delivery of siRNA.

Since gene delivery utilizing non-viral vector is more promising, the polymers that is capable to mimic or show the properties that viral vector vehicles have such as protecting the genomic information, directing the delivery to the target organs and entering the cell membrane are of the interest to the scientists. [7] In the progress of the recent decades, numbers of polymers meeting these requirements were developed, such commonly used polymers include: polyethyleneimine (PEI) (Figure 2), poly(D,L-lactic-co-glycolic acid) (PLGA) (Figure 3), and Chitosan (Figure 4). PEI, PLGA, and chitosan. PEI and chitosan contain amine groups while PLGA has no amine group and have shown scientist their great potentials. However, there are differences between the three polymers regarding the transfection efficiency, toxicity, and other important aspects that greatly impact the versatility of the polymer.

This study aims to explore the applications of the polymers applied in gene delivery, with regard in focusing on the comparison between the common polymers, as well as delving into the parameters that affects the overall transfection efficiency and discuss the future for the polymer applications.

\section{Parameters}

There are factors that affects the evaluation of a certain polymer, mostly including the cytotoxicity, targeting capability, transfection efficiency.

Cytotoxicity measurement is the evaluation of the toxicity of a medical device to the cells of interest. [8] In the case of gene delivery, cytotoxicity study measure of toxicity of polymers in the form of cell viability after a certain amount of time after the polymers being introduced to host cells. Cytotoxicity study involves the most commonly used 3-(4,5-dimethylthia-zol-2-yl)-2,5diphenyltetrazolium bromide (MTT) assay, developed by Mosmann which enable the testing of cell growth rate and toxicity of the cell culture. [8] [9] In gene delivery, cells are cultured and given to contact with the polymers of certain concentration in a certain amount of time such as $24 \mathrm{~h}$. After the contact time, the cells viability associated with such polymer would be compared to other polymer under the same conditions. Transfection efficiency utilizes Luciferase assay, which can be used to obtain a functional relationship of the amount of gene transferred with the luminescence produced by the protein expressed by the cell.

Transfection efficiency in also very crucial, as it dictates the efficiency of such delivery. Transfection is a process of introducing the genetic material into host cells, by methods such as sonoporation and polyplex system. The polyplex containing the hydrophilic gene can enter the hydrophobic cell membrane and introduce the gene by endocytosis where the polyplex enter the cell entirely or by cell fusion where the polyplex fuse with the cell. [10] There are a few factors that affects the transfection efficiency which are considered in the process of developing a polyplex. Such factors include molecular weight, stability, toxicity, and architecture of a polymer. In the study of the effect of molecular weight, galactosylated PEI (Gal-PEI) of different molecular weight along with the same PEI without galactosylation. For PEI, the PEI with molecular weight of $1800 \mathrm{Da}$ is most efficient for transfecting HepG2 cells than PEI of $10,000 \mathrm{Da}$. After galactosylation, $\mathrm{Gal}_{19}-\mathrm{PEI}_{10,000}$ shows the greatest transfection efficiency. In this case, PEI with higher molecular weight is more efficient. However, the opposite can also be observed in the case of transfect HEK293 cells using chitosan, where chitosan oligomers molecular weight of 4.7 to $33 \mathrm{kDa}$. Besides, molecular weight of PEI, the architecture also plays a role in transfection efficiency, where linear PEI is more effective and branched PEI. [11] [12] Stability dictates how long will the polymer be broken down or leave the system. If the polymer and DNA complex is not stable, the gene successfully delivered would be less than the stable complex. Stability of the polyplex can be improved or controlled by modifying the substitution on the backbone, and the chain length of the polymer such as chitosan. One example would be glycosylation which improves the colloidal stability and the stability decreases with the increase in length of the substituent on the chitosan backbone. [13] In addition, toxicity affects how much cells would die in an amount of time. If the transfected cells die, the gene delivery is essentially meaningless as those cells can't express or silence a protein.

Targeting capability also plays a big role, as it enables the complex containing the genetic materials to target the desired cell. Without targeting capability, the genetic material would not be delivered to the target cells, resulting in having no therapeutic effect or causing damage to cells in the worse scenario.

\section{The Application of Polymers in Non- viral Vectors}

The non-viral delivery requires a formation of complex or polyplex between the anionic gene material and a cationic polymer, utilizing the electrostatic interaction. [7] Usually, such cationic polymers contain amine group to facilitate the forming of complex between the polymer and the gene materials, resulting in the transfection rates 
higher than the naked DNA by up to four times. [11] [14] Before polymers were incorporated in the non-viral vectors, cationic lipids were used more frequently. Polymers application are more advantageous in delivery due to better transfection efficiency and other important aspects such as cytotoxicity. [11]

The development of PEI has been a focus since 1995 and Boussif et al. indicated that PEI would be an effective gene transfection agent. There are two structures of PEI being developed which serve the purpose of forming complexes, where one is linear and the other is branched. For complexing DNA and PEI, the linear PEI is superior to the branched PEI under the condition that both are $22 \mathrm{~kg} / \mathrm{mol}$ molecular weight. [7] PEI with both linear and branched architectures are shown to be more effective than cationic lipoplex of DCCholesterol/Dioleoyl

Phosphatidylethanolamine(DCChol/DOPE) for airway delivery in mouse as the luciferase expression assay shows the superior transfection efficiency of PEI. [11] In general, although both architecture of PEI shows superior transfection efficiency, the linear architecture is consistently more advantageous than the branched counterpart. Although PEI is effective as a cationic polymer for delivery, the cytotoxicity has been a major problem, especially cytotoxicity is one of the most critical aspects. Because of the cytotoxicity and the excellent complexing ability, the PEI derivatives were in the development, prompting scientists to make the linkages to be more degradable in body.<smiles>CC(C)(C)CCNC(C)(C)C</smiles>

Figure 2. The structure of Polyethyleneimine (PEI)

Because of the cytotoxicity, PEI is often used along with other polymers such as PLGA. The core forming the complex with the gene material is PLGA with PEI acting as the coating. PLGA shows great properties including biodegradability, mechanical properties, and biocompatibility. In gene delivery, PLGA is presents great targeting capability as well as protecting gene materials safely. [15] [16] Also, PLGA or PLGA derivatives can incorporate with other polymers to enhance the transfection efficiency and decrease cytotoxicity. Transfection efficiency of PLGA/PEI/DNA complex and high molecular weight PEI were compared in green fluorescent protein assay. Human hepatocellular carcinoma cells were cultured for the cytotoxicity analysis. The PLGA/PEI/DNA complex presents high transfection efficiency while showing less cytotoxicity in vitro than PEI with high molecular weight and liposome. [15] Currently, the application of PLGA nanoparticles in the treatment of tumor, utilizing ultrasound, is successful due to the great targeting capability and the transparency in ultrasonography.<smiles>CC(=O)C(C)OC(=O)CO[Tl]</smiles>

Figure 3. The structure of poly(D,L-lactic-co-glycolic acid) (PLGA)

Chitosan also interest scientists due to the potential in gene delivery. Chitosan is a derivative of natural cationic polysaccharide, chitin, with a repeating unit of glucosamine and some degree of acetyl groups present in the repeating units. The degree of deacetylation yields different chitosan derivatives with varying properties including solubility. Since chitosan is a natural polymer, it is biodegradable. More importantly, low cytotoxicity has been observed which is one of the important criteria for considering an application of a certain material in gene delivery. The complex formation of cationic chitosan and anionic DNA decreases the degradation rate of DNA. [17] [18] Chitosan derivatives are also useful and developed in gene delivery. Trimethyl chitosan is used in gene delivery and later modified with polyethylene glycol grafted on for improved transfection efficiency. Besides trimethyl chitosan, chitosan can also be modified by lactose. This lactosylation process increases the targeting capability as the galactose portion of lactose targets the cell membranes that express galactose binding lectin. Lactosylation reduces the surface potential which increase the tendency for aggregating, but also reduce the tendency for forming complex with DNA as the content of lactose increases. [18] In the delivery of targeting HeLa cells with presence of $10 \%$ serum, chitosan shows greater transfection efficiency than PEI. Although PEI still remains the most efficient polymer for gene delivery of common cells such as HepG2 and BNL CL.2, the lower cytotoxicity of lactosylated chitosan/DNA complex is something that PEI application needs to match.

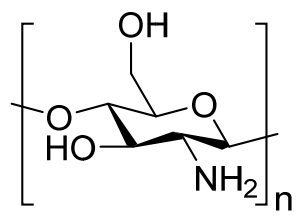

Figure 4. The structure of chitosan

Since there are so many commonly used polymer materials in gene delivery, knowing the advantages and the shortcomings of each polymer is vital for the application, as the cytotoxicity and transfection efficiency play crucial role. In the MTT assay, human liver cancer cell (HepG2) was used for the study of cytotoxicity. The cytotoxicity of corresponding polymers or polymer complex is shown in Table 1. [15] In Table 1, the comparison of PEI/DNA and PEI/PLGA/DNA complex is compared, where the PLGA content decreases cytotoxicity as the cell viability is significantly higher than the PEI/DNA complex. HepG2 cell viability $48 \mathrm{~h}$ post transfection in $37^{\circ} \mathrm{C}$ in humidified and $5 \%$ $\mathrm{CO}_{2}$ incubator. The transition efficiency for 
PEI/PLGA/DNA complex is the highest round $39 \%$ when the nanoparticle (PEI/PLGA) to DNA ratio reaches $6: 1$. In this case, PLGA helps the encapsulation of the gene material.

Table 1. Green Fluorescent protein (GFP) expression in HepG2 with $25 \mathrm{kDa}$ PEI.

\begin{tabular}{|c|c|c|}
\hline Complex & Cell viability (\%) & $\begin{array}{c}\text { GFP positive } \\
\text { cell } \%\end{array}$ \\
\hline PEI & 54 & 17 \\
\hline PLGA/PEI & 90 & up to 39 \\
\hline
\end{tabular}

Chitosan is used in gene delivery in the form of trimethylation. In Table 2 , the cytotoxicity test of TMC with varying trimethylation and PEI/DNA complex with $25 \mathrm{kDA}$ linear PEI (PEI: DNA 10: $1 \mathrm{w} / \mathrm{w}$ ) in human breast epithelial cells (MCF-7) is shown. In this comparison, trimethyl chitosan (TMC) with varying degree of trimethylation and linear PEI. TMC with varying degree of trimethylation shows greater cell viability in human breast epithelial cells (MCF-7) while the transfection efficiency of TMC is higher than PEI. When using the in African green monkey kidney fibroblast like cells (COS-7), the transfection efficiency of PEI is higher than any of the TMC and TMO (trimethyl chitosan oligomers). [9] Trimethylated chitosan oligosaccharide of 44\% (TMO 44) is presenting a great transfection efficiency. The transfection efficiency of TMO 44 is better than TMC of varying trimethylation in gene delivery involves COS-7 cells and slightly worse than TMC 57 and TMC 93 in MCF-7 cell.

Table 2. Cell viability comparison. between different trimethylation percentage and PEI.

\begin{tabular}{|c|c|c|}
\hline Complex & $\begin{array}{c}\text { Cell viability } \\
(\%)\end{array}$ & $\begin{array}{c}\text { Luminescence } \\
\text { (RLU mg-1) }\end{array}$ \\
\hline TMC 36/DNA & 83 & $1 \times 10^{\wedge} 1$ \\
\hline TMC 57/DNA & 80 & $1 \times 10^{\wedge} 3$ \\
\hline TMC 76/DNA & 95 & $1 \times 10^{\wedge} 0.4$ \\
\hline TMC 93/DNA & 80 & $1 \times 10^{\wedge} 3.3$ \\
\hline PEI/DNA & 56 & $1 \times 10^{\wedge} 1.5$ \\
\hline
\end{tabular}

PEI, PLGA and chitosan all have great potentials and applications in gene delivery but not all have the same advantages and shortcomings which is summarized in Table 3. [4] [7] [11] [19] [20]

Table 3. The comparison of PEI, PLGA, and chitosan.

\begin{tabular}{|l|l|l|l|}
\hline & Applications & Advantages & Shortcomings \\
\hline PEI & $\begin{array}{l}\text { Polyplex } \\
\text { with siRNA } \\
\text { or DNA }\end{array}$ & $\begin{array}{l}\text { - Good transfec- } \\
\text { tion efficiency. } \\
\text { - Excellent com- } \\
\text { plexing ability }\end{array}$ & $\begin{array}{l}\text { - High cyto- } \\
\text { toxicity. }\end{array}$ \\
\hline PLGA & $\begin{array}{l}\text { Polyplex } \\
\text { with siRNA } \\
\text { or DNA }\end{array}$ & $\begin{array}{l}\text { - Able to incor- } \\
\text { porate into PEI } \\
\text { polyplex to in- } \\
\text { crease transfec- } \\
\text { tion efficiency } \\
\text { and decrease }\end{array}$ & $\begin{array}{l}\text { - Used alone } \\
\text { leads to } \\
\text { rapid elim- } \\
\text { ination by } \\
\text { immune }\end{array}$ \\
\hline
\end{tabular}

\begin{tabular}{|c|c|c|c|}
\hline & & $\begin{array}{l}\text { cytotoxicity. } \\
\text { - Great targeting } \\
\text { capability. } \\
\text { - Safely protect- } \\
\text { ing gene mate- } \\
\text { rials }\end{array}$ & system. \\
\hline Chitosan & $\begin{array}{l}\text { Polyplex } \\
\text { with siRNA } \\
\text { or DNA }\end{array}$ & $\begin{array}{l}\text { - Improved } \\
\text { transfection ef- } \\
\text { ficiency with } \\
\text { polyethylene } \\
\text { glycol grafted } \\
\text { on. } \\
\text { - Availability. } \\
\text { - Low cytotoxi- } \\
\text { city } \\
\text { - Biodegradable } \\
\text { and compatible }\end{array}$ & $\begin{array}{l}\text { - Complex } \\
\text { forming } \\
\text { capability }\end{array}$ \\
\hline
\end{tabular}

There are other polymers, such as poly(propylene oxide) (PPO), poly(ethylene oxide) (PEO), and poly dimethylaminoethylmethacrylate (PDMAEMA), are used in gene delivery and present significance. PPO and PEO are used an ABA type block copolymer named Pluronic and poloxamer as hydrophobic core, approved by FDA in biomedical applications. PDMAEMA is used as art of hydrophilic shell for increasing transfection efficiency. [4]

\section{The Future of the Polymers in Gene Delivery}

Gene delivery is a particularly efficient and novel way of treating diseases that are caused by defects in genes that involve expressing an extra or lacking a certain protein. Gene delivery has been increasingly focused and developed with numerous successful treatments for patients. However, when the parameters of the genepolymer complex are not well balanced such as the material having high transfection efficiency and cytotoxicity at the same time, the therapeutic effects of polymer or complex are not as efficient. In some case, such imbalance causes no therapeutic effects, but in the worst scenario, causing fatal damages to cells due to cytotoxicity, such as PEI having high transfection efficiency and cytotoxicity at the same time. Another drawback that has a great impact would be the off-target effects. If the materials utilized has low targeting capability, the gene would not be delivered to the desired target cells, causing no therapeutic and other unexpected effects, including interferon response and toxic phenotypes impairing patients' health. [21]

Since cytotoxicity is one of the most important aspects in gene delivery, the reducing of cytotoxicity is crucial for gene delivery and health concerns. As discussed in the previous sections, linear and branched PEI are excellent in transfecting but resulting in high cytotoxicity compared to most of the commonly used polymers. To reduce cytotoxicity and maintain transfection efficiency, modifying PEI or introducing other polymers such as PLGA into the complex 
formulation, greatly reduces the cytotoxicity as shown in Table 1. Alternatively, the other route is also effective, involving choosing or developing materials with low cytotoxicity and modify the polymer towards a higher transfection efficiency. The development of chitosan is similar to such alternative route, having modifications such as trimethylated chitosan improves transfection efficiency. As an example, in reaction scheme shown in Figure 5, chitosan can be modified to chitosan derivative, O-carboxymethyl chitosan (OCMCh). Modifying from OCMCh, HPOCP consisting OCMCh/branched $\mathrm{PEI} /$ targeting ligand (specific to $\mathrm{HER}_{2}$ site) can be synthesized, presenting anticancer therapeutic effects with significantly lower cytotoxicity, higher transfection efficiency compared to PEI. The ligand, labeled as $\mathrm{HER}_{2}$ in the reaction scheme is site specific to human epidermal growth factor receptor $2\left(\mathrm{HER}_{2}\right)$, which allows the polyplex to have an improved delivery in terms of targeting capability and specificity to $\mathrm{HER}_{2}$. PEI can be grafted onto the O-carboxymethyl chitosan (OCMPEI), also presenting significantly less cytotoxicity. [22] Another important polymer modifications involves hydrophobic modification. Such modification involves the control of the length of the side chains on the polymer backbone, improving transfection efficiency by providing better cellular, colloidal stability and reducing cytotoxicity. [23] Chitosan can be modified to obtain better hydrophobicity, utilizing ring opening reaction with deoxycholic acid, which proves to be efficient in gene delivery. [24]

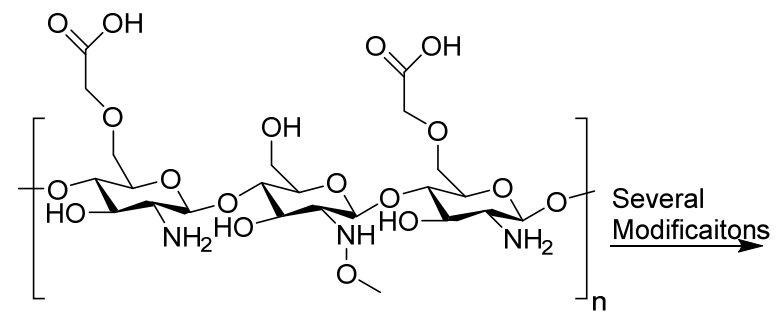

OCMCh

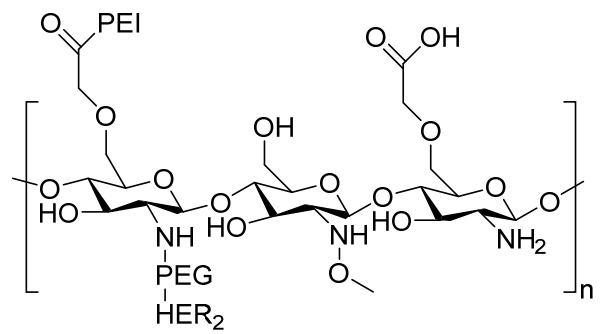

HPOCP

Figure 5. Modification of O-carboxymethyl chitosan with site targeting ligand.

Off-target effects are one of the obstacles that waits for solving as those effects can be dangerous and indistinguishable from on-target silencing. [25] For siRNA delivery, off-target effects can be reduced, utilizing local alignment algorithms, including BLAST and Smith-Waterman. Smith-Waterman algorithms makes predictions base on simulations of the off-targets with magnitudes 1 to 2 orders higher than true value. [21]
Besides the two algorithms, the concentration and modifications of siRNA can play a role in minimizing off-target affects. As the off-target effects depends on concentration, lowering the concentration reduces or even eliminates off-target effects, but at the expense of gene silencing effects. Chemical modification on siRNA proves to be useful for eliminating up to $80 \%$ off-target effects. [25] Modification of siRNA includes 2'-Omethyl modification, adding a methyl group to the $\mathrm{C} 2$ position of a ribose portion of the nucleoside, to a specific seed region, reducing off-target effect without compromising silencing capability. [25]With the help of the improving of complex formation between the chemically modified gene material and the polymers with great targeting capability, such as modifying the polymer with $\mathrm{HER}_{2}$ targeting ligand for delivery targeting $\mathrm{HER}_{2}$, the targeting specificity can be greatly improved.

As discussed, gene delivery requires fine-tuning modifications on the polymer such as PEI, chitosan, and PLGA to show therapeutic effects such as expressing or silencing a particular gene, in regard of cytotoxicity, transfection efficiency, and minimizing off-target effects. The future of the development of the polymer complex of the delivery would be focusing on the fine tuning of the polymers in current use and the trials of the new polymer materials for gene delivery.

\section{Conclusion}

In this study, gene delivery or therapy is discussed regarding the two different vectors, the various important parameters in consideration when developing a delivery material, and the polymers in current applications, as well as some of the limitations and the strategies for improvements. As an example, the application of polymer in cellular delivery of siRNA is summarized and illustrated in Figure 5. Gene delivery is still a very important and efficient therapy method for treating genetic diseases, and is made possible by the genius modifications on the existing polymers, the trails of different composition of each component in a polymer complex, and the numerous endeavors invested. In the near future, the application of gene delivery would be capable and more efficient in treating the untreatable genetic diseases from the past and the polymers in the application would be increasingly efficient and stable in transfecting cells.

\section{References}

1. Herskovic, A.; Martz, K.; Al-Sarraf, M.; Leichman, L.; Brindle, J.; Vaitkevicius, V.; Cooper, J.; Byhardt, R.; Davis, L.; Emami, B., Combined chemotherapy and radiotherapy compared with radiotherapy alone in patients with cancer of the esophagus. New England Journal of Medicine 1992, 326 (24), 1593-1598.

2. Rosenberg, S. A.; Aebersold, P.; Cornetta, K.; Kasid, A.; Morgan, R. A.; Moen, R.; Karson, E. M.; Lotze, M. T.; Yang, J. C.; Topalian, S. L., 
Gene transfer into humans-immunotherapy of patients with advanced melanoma, using tumorinfiltrating lymphocytes modified by retroviral gene transduction. New England Journal of Medicine 1990, 323 (9), 570-578.

3. Cotrim, A. P.; Baum, B. J., Gene therapy: some history, applications, problems, and prospects. Toxicologic pathology 2008, 36 (1), 97-103.

4. Marzbali, M. Y.; Khosroushahi, A. Y., Polymeric micelles as mighty nanocarriers for cancer gene therapy: a review. Cancer chemotherapy and pharmacology 2017, 79 (4), 637-649.

5. Sung, Y.; Kim, S., Recent advances in the development of gene delivery systems. Biomaterials research 2019, 23 (1), 8.

6. Lundstrom, K., Viral vectors in gene therapy. Diseases 2018, 6 (2), 42.

7. Thomas, T.; Tajmir-Riahi, H.-A.; Pillai, C., Biodegradable polymers for gene delivery. Molecules 2019, 24 (20), 3744.

8. Li, W.; Zhou, J.; Xu, Y., Study of the in vitro cytotoxicity testing of medical devices. Biomedical reports 2015, 3 (5), 617-620.

9. Kean, T.; Roth, S.; Thanou, M., Trimethylated chitosans as non-viral gene delivery vectors: cytotoxicity and transfection efficiency. Journal of Controlled Release 2005, 103 (3), 643-653.

10. Ziello, J. E.; Huang, Y.; Jovin, I. S., Cellular endocytosis and gene delivery. Molecular Medicine 2010, 16 (5-6), 222-229.

11. Wiseman, J.; Goddard, C.; McLelland, D.; Colledge, W., A comparison of linear and branched polyethylenimine (PEI) with DCChol/DOPE liposomes for gene delivery to epithelial cells in vitro and in vivo. Gene therapy 2003, 10 (19), 16541662.

12. Morimoto, K.; Nishikawa, M.; Kawakami, S.; Nakano, T.; Hattori, Y.; Fumoto, S.; Yamashita, F.; Hashida, M., Molecular weight-dependent gene transfection activity of unmodified and galactosylated polyethyleneimine on hepatoma cells and mouse liver. Molecular Therapy 2003, 7 (2), 254-261.

13. Strand, S. P.; Lelu, S.; Reitan, N. K.; de Lange Davies, C.; Artursson, P.; Vårum, K. M., Molecular design of chitosan gene delivery systems with an optimized balance between polyplex stability and polyplex unpacking. Biomaterials 2010, 31 (5), 975987.

14. Dincer, S.; Türk, M.; Pişkin, E., Intelligent polymers as nonviral vectors. Gene therapy $\mathbf{2 0 0 5}$, 12 (1), S139-S145.

15. Liang, G. F.; Zhu, Y. L.; Sun, B.; Hu, F. H.; Tian, T.; Li, S. C.; Xiao, Z. D., PLGA-based gene delivering nanoparticle enhance suppression effect of miRNA in HePG2 cells. Nanoscale research letters 2011, 6 (1), 447.
16. Figueiredo, M.; Esenaliev, R., PLGA nanoparticles for ultrasound-mediated gene delivery to solid tumors. Journal of drug delivery 2012, 2012.

17. Saranya, N.; Moorthi, A.; Saravanan, S.; Devi, M. P.; Selvamurugan, N., Chitosan and its derivatives for gene delivery. International journal of biological macromolecules 2011, 48 (2), 234-238.

18. Erbacher, P.; Zou, S.; Bettinger, T.; Steffan, A.M.; Remy, J.-S., Chitosan-based vector/DNA complexes for gene delivery: biophysical characteristics and transfection ability. Pharmaceutical research 1998, 15 (9), 1332-1339.

19. Bose, R. J.; Lee, S.-H.; Park, H., Lipid-based surface engineering of PLGA nanoparticles for drug and gene delivery applications. Biomaterials Research 2016, 20 (1), 34.

20. Patil, Y.; Panyam, J., Polymeric nanoparticles for siRNA delivery and gene silencing. International journal of pharmaceutics 2009, 367 (1-2), 195-203.

21. Scientific, T. F., Off-Target Effects: Disturbing the silence of RNA interference (RNAi). Thermo Fisher Scientific Teach review 2010.

22. Nam, J.-P.; Nah, J.-W., Target gene delivery from targeting ligand conjugated chitosan-PEI copolymer for cancer therapy. Carbohydrate polymers 2016, $135,153-161$.

23. Chen, C.-K.; Huang, P.-K.; Law, W.-C.; Chu, C.H.; Chen, N.-T.; Lo, L.-W., Biodegradable polymers for gene-delivery applications. International journal of nanomedicine 2020, 15, 2131.

24. Liu, Z.; Zhang, Z.; Zhou, C.; Jiao, Y., Hydrophobic modifications of cationic polymers for gene delivery. Progress in Polymer Science 2010, 35 (9), 11441162.

25. Jackson, A. L.; Burchard, J.; Leake, D.; Reynolds, A.; Schelter, J.; Guo, J.; Johnson, J. M.; Lim, L.; Karpilow, J.; Nichols, K., Position-specific chemical modification of siRNAs reduces "off-target" transcript silencing. Rna 2006, 12 (7), 1197-1205. 